\title{
CARACTERIZACIÓN DE LOS SISTEMAS DE ACTUACIÓN PARA VEHÍCULOS ALTAMENTE AUTOMATIZADOS
}

\author{
Joseba Sarabia $^{a}$, Jose Angel Matute ${ }^{a}$, Asier Zubizarreta ${ }^{b}$. \\ ${ }^{a}$ Tecnalia Research \& Innovation, División de Industria y Transporte, España \\ ${ }^{b}$ Departamento de Ingeniería de Sistemas y Automática, Universidad del País Vasco (UPV/EHU) \\ Facultad de Ingniería de Bilbao, España
}

\section{Resumen}

Los modelos de simulación son importantes para la investigación y desarrollo de estrategias de decisión y control en vehículos automatizados. Las plataformas de pruebas reales se obtienen generalmente mediante la modificación de vehículos comerciales, donde robots o actuadores electromecánicos son fijados a los elementos de conducción del vehículo como el volante, pedales de freno y aceleración. Diferencias significativas durante el ajuste de las ganancias en el control de alto nivel entre simulaciones y dichas plataformas reales pueden deberse principalmente a un modelo impreciso de los actuadores. En este trabajo, se presenta una revisión del estado del arte sobre técnicas para el control de bajo nivel en diferentes plataformas utilizadas tanto en lo académico como en la industria. Adicionalmente, se muestra la metodología utilizada para el modelado de actuadores realizando pruebas a lazo abierto sobre una plataforma real para pruebas de conducción automatizada. Finalmente, se presenta una comparativa entre el comportamiento de los actuadores modelados y los reales durante un recorrido del vehículo en modo automático ejecutando una ruta predefinida, evidenciando la fiabilidad de los modelos propuestos y la metodología utilizada para su obtención.

Palabras clave: vehículo autónomo, identificación de parámetros, modelado, simulación, actuador electromecánico, automoción.

\section{INTRODUCCIÓN}

Para entender el largo recorrido que conlleva la consecución del vehículo autónomo (VA), es necesario atender a la complejidad de la estructura de los subsistemas del mismo. Como se ve en la Fig. 1, esta estructura está dividida en diversas capas [1]:

En primer lugar, la capa de sensorización. Esta capa es la encargada de recoger los datos provenientes del entorno del vehículo. Está compuesta de todos los sensores necesarios para posteriormente obtener su localización y orientación.

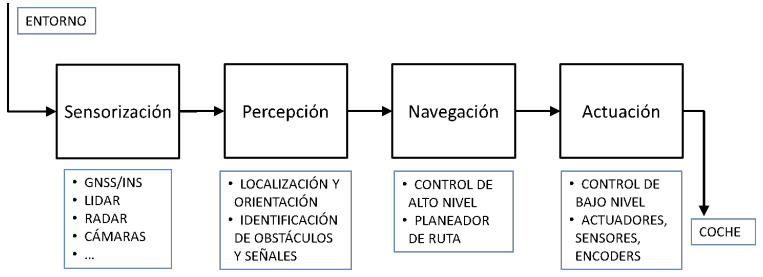

Figura 1: Estructura de control de un vehículo autónomo

A continuación, viene la capa de percepción. Esta interfaz procesa los datos obtenidos de la capa de sensorización. Es decir, procesa los datos de localización y orientación, tanto del vehículo como de la carretera y los obstáculos de alrededor.

Después, viene la capa de navegación. Valiéndose de la información del entorno y la ruta deseada, traza la trayectoria instantánea que el automóvil va a tomar. Aquí se ubica el control de alto nivel del vehículo, donde se deberá escoger el algoritmo de toma de decisiones.

Finalmente, está la capa de actuación. Su función consiste en traducir la trayectoria deseada en comandos. Estos comandos se transmiten a los sistemas de actuación del vehículo, para que este se desplace de forma deseada. Esta capa cuenta con un sistema de control independiente de la capa superior. Es aquí donde se centra este trabajo.

Cuando se aborda la automatización de un vehículo, es necesario sustituir sus controles manuales (volante y pedales de acelerador y freno) por un sistema electromecánico que permita seguir las consignas del controlador de alto nivel. A la hora de abordar esta problemática, existen tres principales alternativas: a) Sistemas adaptados que actúan externamente sobre los actuadores del vehículo, b) sistemas que utilizan los actuadores internos del vehículo, y c) vehículos implícitamente diseñados para ser autónomos con todos sus sistemas integrados.

El mayor exponente del primer caso es el sistema EMC de AEVIT [2]. Se trata de un modelo comercial patentado y aprobado para su uso en carretera. Consta un módulo para el control de los 
actuadores, un servomotor para cada sistema de actuación (freno, acelerador y volante), un sistema de alimentación propio, y un HMI para su manejo. Este sistema fue el más utilizado en el DARPA Urban Challenge 2007 [3], el cual está considerado como el primer gran paso en el recorrido del vehículo autónomo.

En segundo lugar, desde 2014 se ha extendido el uso de los sistemas drive-by-wire [4]. Utilizando los sistemas propios de actuación del vehículo instalados en el control de tracción o el control de estabilidad, es posible controlar el vehículo para tareas de conducción automatizada. Para ello, se conecta el ordenador a la red CAN del vehículo, evitando incluir actuadores externos.

Finalmente, existen nuevos prototipos diseñados para ser puramente autónomos. Estos no cuentan con pedales ni volante, si no que se trata de vehículos alternativos que apuestan por una autonomía total [5].

Actualmente, existen numerosos prototipos de VA, que cuentan con una de las arquitecturas mencionadas, y que están en la etapa de validación. Uno de los principales inconvenientes de esa etapa es el gran coste económico y temporal que supone probar un prototipo en carretera para certificar su correcto funcionamiento. Por ello, una de las principales líneas de investigación está enfocada en obtener el modelo dinámico del vehículo, para que éste sea probado en simuladores, y así sustituir parte de las pruebas en carretera.

Por ese motivo, en este documento se presenta el modo de obtención de los modelos dinámicos de los sistemas de frenado y de dirección, los cuales formarán parte del simulador del vehículo completo desarrollado por Tecnalia denominado Dynacar. Gracias a este simulador, se podrá obtener una respuesta lo más fiable posible del modo en el que actuaría el vehículo real. De modo que se facilitará el desarrollo de otras líneas de investigación en un entorno seguro.

Para ello, este documento cuenta con la siguiente distribución. La Sección 2 detalla las plataformas de pruebas para vehículos autónomos desarrollados por Tecnalia, Bernardo y Ruperta. En la Sección 3 se describe la metodología desarrollada para la obtención de los modelos dinámicos diseñados para los sistemas de freno y de dirección. En la Sección 4 se muestran los resultados obtenidos a partir de dichos modelos. Por último, se muestran las conclusiones de este proyecto.

\section{PLATAFORMA DE PRUEBAS BERNARDO Y RUPERTA}

El Grupo de Conducción Automatizada de Tecnalia Research and Innovation está desarrollando una plataforma de investigación para el desarrollo del VA. Para ello, cuenta con dos Renault Twizy E80, como se muestra en la Fig. 2, los proyectos Bernardo y Ruperta, dos cuatricíclos eléctricos biplaza de baja potencia.

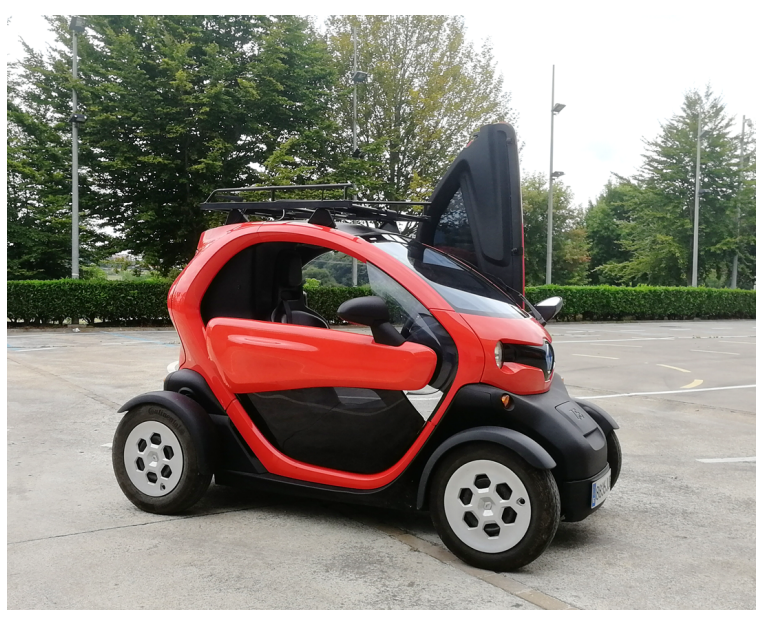

Figura 2: Renault Twizy

Actualmente, ambos vehículos cuentan con el mismo conjunto de dispositivos. Su esquema de control es el mostrado en la Fig. 3. Tal y como se ve, se han incorporado dos actuadores externos al sistema de dirección y de freno, mientras que la consigna de aceleración se puede enviar desde la ECU haciendo un bypass el acelerador y enviando las tramas correspondientes por el bus CAN del vehículo.

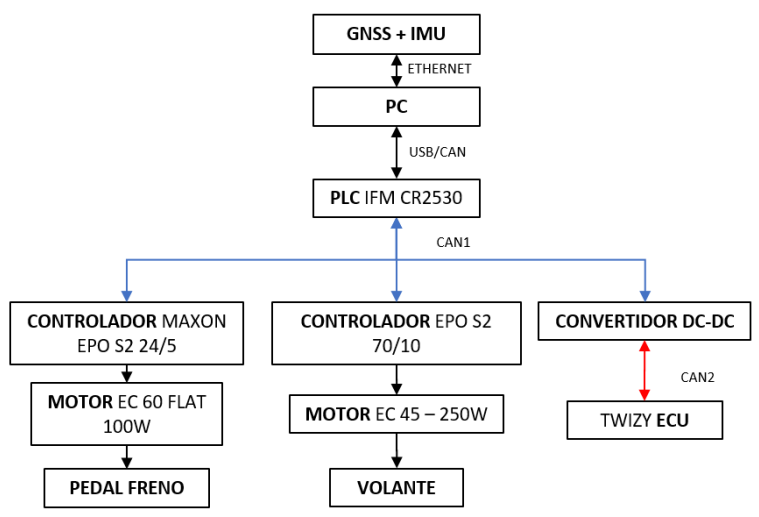

Figura 3: Esquema de control de bajo nivel

El freno está controlado mediante un conjunto de controlador (Maxon EPO S2), motor (Maxon EC 60 Flat) y sensor ( Encoder Mile 512-4096 CPT). El motor actúa unidireccionalmente sobre el freno mediante una cadena. La estrategia de control del 
motor se realiza en el controlador Maxon, el cual recibe del PLC vía CANBUS la señal de referencia, y del encoder la señal de la posición en la que se encuentra.

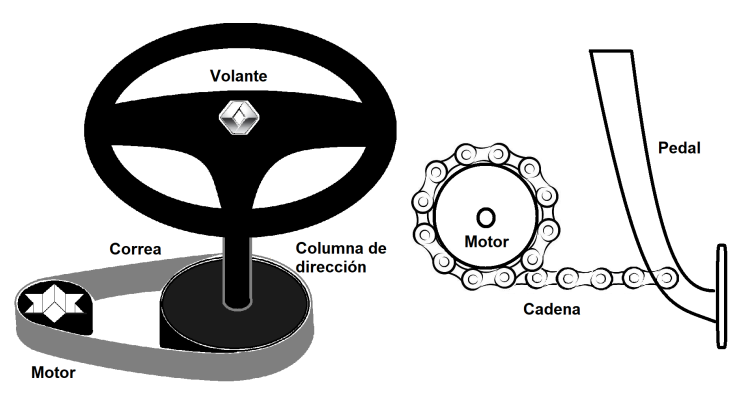

Figura 4: Diagrama de los actuadores

Para el control automático de la dirección se ha instalado un motor en paralelo a la columna de dirección, unido a esta mediante una correa, como se muestra en la Fig. 4. Se trata de un sistema similar al del freno, con un controlador (Maxon EPO S2), motor Maxon EC 45) y doble sensor (Encoder Posital UCD - SRA1G y Held 9140500 CPT). A pesar de la similitud estructural, su punto de funcionamiento es muy distinto. Esto se debe a que el freno debe mostrar una respuesta rápida, mientras que la dirección debe ir limitada acorde con los estándares de giro del vehículo.

Cuadro 1: Parámetros de los motores.

\begin{tabular}{c|cc} 
& Freno & Dirección \\
\hline Modelo & EC 60 Flat & EPO S2 \\
\hline Resistencia $[\mathrm{m} \Omega]$ & 307 & 143 \\
Reactancia $[\mathrm{mH}]$ & 0,188 & 0,0565 \\
Const. de par $[\mathrm{mNm} / \mathrm{A}]$ & 53,4 & 26,3 \\
Const. de t mecánica $[\mathrm{ms}]$ & 13 & 4,43 \\
Inercia del rotor $\left[\mathrm{gcm}^{2}\right]$ & 1210 & 209
\end{tabular}

\section{METODOLOGÍA DE MODELADO DEL SISTEMA DE ACTUACIÓN}

Los modelos de los actuadores se han obtenido mediante dos metodologías diferentes. En primer lugar, el modelo del freno se ha obtenido directamente a través de su entrada y respuesta, sin considerar la planta. Es decir, con los datos sustraídos de las curvas de la Fig. 5. En segundo lugar, el modelo de la dirección se ha obtenido teniendo en cuenta su respuesta correspondiente (Fig. 7) y, además, las propiedades físicas de su sistema. Para ello, se han realizado varias pruebas atacando el sistema con señales escalón de diferente amplitud, a través de las cuales se han obtenido unos parámetros medios como el retraso de la señal, el tiempo de establecimiento y el error estacionario. Dichos parámetros se muestran en la tabla 2 .

El tiempo de retraso $t_{d}$ es el cómputo total de los tiempos de comunicación entre el ordenador y los actuadores, y el tiempo que estos tardan en actuar. El tiempo de establecimiento $t_{\text {est }}$ del sistema de frenado tiene una media de $166 \mathrm{~ms}$, mientras que el del sistema de dirección es mucho más lento y variable. Por un lado, su velocidad debe estar limitada por motivos de seguridad, para evitar giros bruscos. Por el otro, su variabilidad depende de la amplitud de giro. El sistema genera un sobre-impulso en giros amplios, lo que retrasa la respuesta final. El error estacionario en el sistema de frenado es nulo, mientras que en el sistema de existe una ligera desviación proporcional a la señal de entrada $u$.

Para asegurar la veracidad de las pruebas, se han tomado varias muestras de la respuesta de los actuadores, y se ha realizado un cálculo de la precisión y de la repetibilidad, obteniendo unos valores por encima del $95 \%$. Por lo tanto, se tomarán estos datos como una representación fiable del sistema.

Cuadro 2: Parámetros medios de los sistemas de actuación sobre el freno y la dirección.

\begin{tabular}{c|cc} 
& Freno & Dirección \\
\hline$t_{d}[m s]$ & 88 & 81 \\
$t_{\text {est }}[\mathrm{ms}]$ & 166 & $1750-3500$ \\
$e_{\text {est }}[\mathrm{ms}]$ & 0 & $1,016 u$
\end{tabular}

\subsection{MODELADO DEL FRENO}

El sistema de control del freno se rige por las señales de posición, velocidad e intensidad que le provienen del actuador. Dichas señales se procesan en el controlador, en un lazo y con unos parámetros de control desconocidos. Por ello, el modelado se realiza utilizando exclusivamente la respuesta del sistema y la señal de entrada ofrecida por el PLC, como se muestra en la Fig. 3. Estas señales se muestran en la Fig. 5, siendo la verde la referencia y la azul la salida.

Como se puede observar, la dinámica del sistema de frenado es muy similar a la de un modelo de orden superior sobre-amortiguado. Nótese que los datos aquí introducidos abarcan toda el rango de funcionamiento del pedal, desde un frenado ligero hasta el frenado más brusco. Esto se representa en la Fig. 5, donde las señales están normalizadas en tanto por uno del rango total (de cero a uno).

Utilizando los parámetros de la tabla 2 y usando el System Identification Toolbox de Matlab se han creado diversos tipos de modelos, de los cuales el 


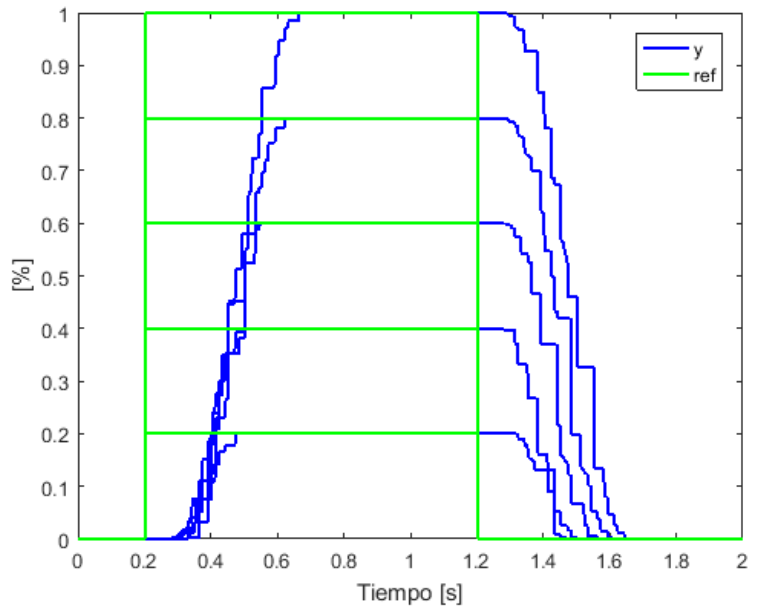

Figura 5: Respuesta del sistema de frenado

más satisfactorio ha resultado ser un modelo de función de transferencia de tercer orden.

$$
G(s)=\frac{0,0265 s^{2}+40,3 s+20358}{s^{3}+52,7 s^{2}+927 s-1,32 \cdot 10^{6}}
$$

Discretizado con un periodo de muestreo de $2 \mathrm{~ms}$.

$$
G(z)=\frac{1,541 \cdot 10^{-4} z^{-1}}{1-2,87 z^{-1}+2,76 z^{-2}-0,9 z^{-3}}
$$

Una vez obtenido el modelo, se le ha añadido un retraso y un limitador de pendiente (rate limiter). Este segundo evita que el actuador alcance unas velocidades no aptas para su sistema. El resultado es un modelo de lazo abierto como el que se muestra en la Fig. 6.

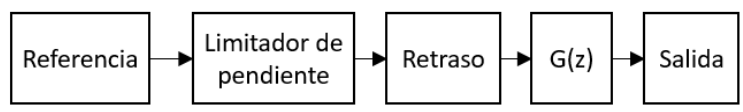

Figura 6: Lazo de control del modelo de frenado

Para la validación, Bernardo ha llevado a cabo un recorrido predefinido en un circuito privado. Posteriormente, se han simulado los actuadores con la señal de referencia ejecutada en dicho circuito, y se han comparado los resultados de los actuadores reales con los resultados de los modelos. La validación se muestra en el Apartado 4.2.

\subsection{MODELADO DE LA DIRECCIÓN}

Para diseñar la dinámica del sistema de dirección, se ha modelado el sistema a partir del motor que actúa sobre el eje de dirección. La dinámica del conjunto motor-columna de dirección está principalmente marcada por la del motor, despreciándose los efectos de inercia de la columna. Por lo tanto, modelando únicamente el motor, es posible obtener la dinámica general del sistema. Para ello, se ha utilizado la expresión de un motor brushless [6], donde la entrada es la señal de tensión introducida al motor, y la salida la velocidad de giro:

$$
G(s)=\frac{\frac{1}{K_{e}}}{\tau_{m} \tau_{e} s^{2}+\tau_{m} s+1}
$$

Dichos parámetros se han cogido de la tabla 1 , siendo $K_{e}$ la constante eléctrica del motor, $\tau_{m}$ la constante de tiempo mecánica y $\tau_{e}$ la constante de tiempo eléctrica.

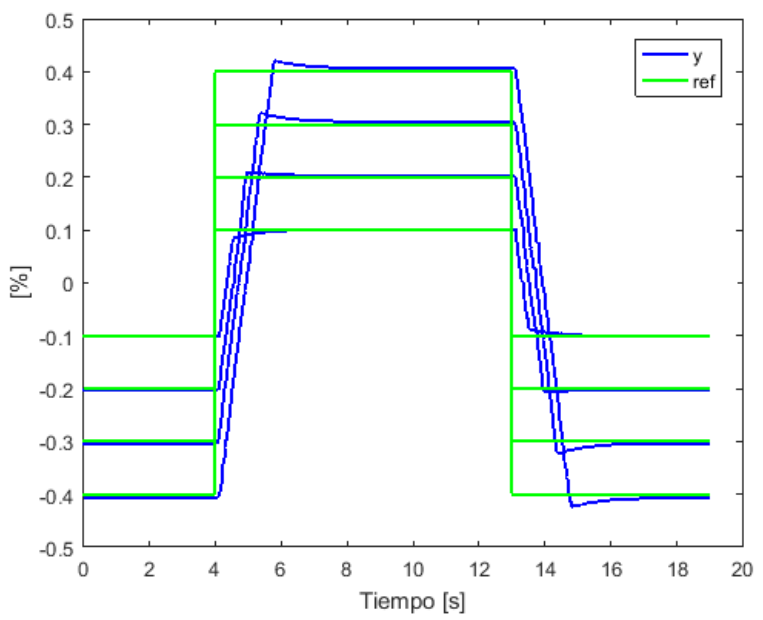

Figura 7: Respuesta del sistema de dirección

Una vez obtenida la función de transferencia, se ha discretizado, para adaptarla al tiempo de muestreo en el que funciona el el sistema real, con un valor de $2 m s$, como se muestra en la ecuación (4).

$$
G(z)=\frac{23,32 z+4,71}{z^{2}-0,41 z+0,0063}
$$

Los parámetros de la tabla 2 se han utilizado para marcar el retardo y el error estacionario de la respuesta. Los parámetros del controlador se han obtenido mediante el PID tuner de Matlab, ya que ofrece la ventaja de calcularlos de forma gráfica. Esta característica permite imitar la respuesta real del sistema. Este mismo procedimiento se podría seguir para el crear un modelo físico del freno, utilizando los parámetros del motor del freno de la tabla 1 .

Llegado a este punto, el principal inconveniente para modelar este sistema es simular su no linealidad. La respuesta del sistema real se ha obtenido a partir de 4 pulsos de entre el $10 \%$ y el $40 \%$ de giro del volante, en ambos sentidos (sentido horario positivo y anti-horario negativo). Como se puede observar en la Fig. 7, para giros leves (amplitud 0.1) la señal tiene una dinámica tendente a un primer orden, mientras que las señales superiores muestran una dinámica diferente. Esta no 
linealidad del sistema de giro está causada por el sistema de control del actuador. Este cuenta con un limitador de velocidad, por lo que, a partir de cierta amplitud de giro, el volante gira siempre a la misma velocidad. Esto es visible en la Fig. 7, dado que todas las señales reales tienen la misma pendiente. Es decir, que se está saturando la señal. Para simular esta no linealidad, se ha insertado un rate limiter al sistema, como se ve en la Fig. 8. Dado que la respuesta de la planta debe ser la posición de giro del volante y no su velocidad, se le ha añadido acción integral a esta señal.

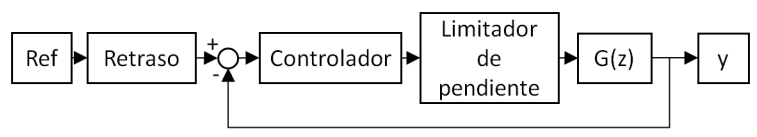

Figura 8: Modelo del sistema de dirección

Debido a la diferencia entre la señal emitida por el controlador y la filtrada por el rate limiter, la acción integral del controlador puede acumular la señal de error, causando un problema de windup, por el cual se dispara el sobre-impulso, y obteniendose en el modelo una respuesta muy desproporcionada y diferente a la del sistema real. Para reducir este efecto, se ha configurado el controlador con un método anti-windup. Este método compara la señal que se le envía al sistema con la señal filtrada por el limitador de pendiente. Esta diferencia es realimentanda al integrador del controlador, donde se multiplica por una ganancia y se resta a la señal del integrador. De este modo, se evita el cúmulo, y no hay tal sobre-impulso, ajustándose así la respuesta del modelo a la respuesta real.

\section{VALIDACIÓN}

En este apartado se muestran los resultados de la validación realizada para los modelos anteriormente detallados. Para ello, se han realizado dos tipos de pruebas: a) pruebas estáticas, en las que se ha probado el funcionamiento de los modelos en parado; y b) pruebas dinámicas, en las que se ha simulado un circuito predefinido, para poner a prueba los modelos en movimiento.

En la prueba estática, se ha realizado una serie de repeticiones comparando ambos resultados, de los cuales se ha calculado el error haciendo una media entre todos ellos, lo cual se muestra en los cuadros 3 y 4 . Además, se ha marcado una zona de confiabilidad con puntos negros alrededor de los datos de los resultados, visible en las figuras 9 y 10 . Esta zona marca la región en la cual un resultado tiene un margen de error igual o menor al $5 \%$. Es decir, que la pertenencia de los datos del modelo respecto a este rango indica que su validez. En cuanto a la prueba dinámica, se muestran los datos de un recorrido realizado. Por lo que los datos del error se muestran de forma gráfica.

\subsection{PRUEBA ESTÁTICA}

Para este caso, se ha realizado una prueba idéntica a la utilizada para la obtención de datos a través de los cuales se desarrollaron los modelos. Para el caso del freno, como se puede ver en la Fig. 9, los datos del modelo se ajustan adecuadamente a los datos reales.

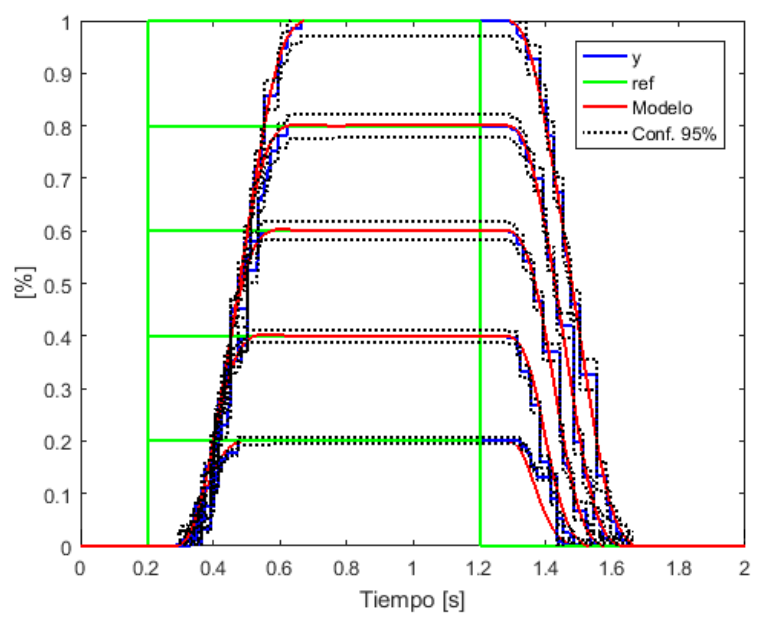

Figura 9: Modelo del sistema de frenado frente a pulso rectangular

El resultado de la precisión para cada uno de los pulsos mostrados en la Fig. 9 se muestra en la tabla 3. La precisión se ha calculado como la unidad menos el error relativo promediado. Para estas pruebas, se justifica el empleo del error relativo promedio dado que el objetivo es mostrar el error en la tendencia de los modelos, mientras que el error absoluto se muestra en el error estacionario. En el cálculo del error relativo promedio, se ha tenido en cuenta únicamente el periodo desde el instante de subida hasta el tiempo de establecimiento. En la tercera columna se muestra S, la desviación estándar del error para cada pulso mostrándose una dispersión del error muy baja.

\begin{tabular}{|c|c|c}
\multicolumn{3}{c}{ Cuadro 3: Resultados del modelo del freno } \\
Amplitud [\%] & Precisión [\%] & S \\
\hline 20 & 96.75 & 0.070 \\
40 & 98.01 & 0.038 \\
60 & 97.94 & 0.039 \\
80 & 97.91 & 0.036 \\
100 & 98.21 & 0.027
\end{tabular}

En el caso del modelo de la dirección, los resulta- 
dos son visibles en la Fig. 10. El modelo reproduce de forma fiel la dinámica del motor. También es perceptible la representación de la no linealidad del sistema en el pulso más bajo, donde no hay sobre-impulso.

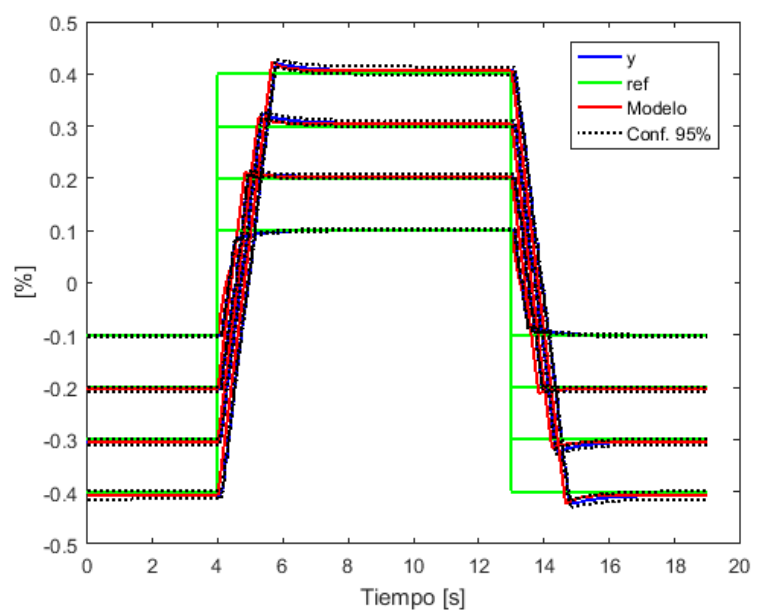

Figura 10: Modelo del sistema de dirección frente a pulso rectangular

En cuanto al error del modelo, cuenta con una precisión no inferior al $93 \%$ para cada pulso. Además, la desviación típica del error en este caso también es muy baja, ratificándose así su validez.

Cuadro 4: Resultados del modelo de la dirección

\begin{tabular}{c|c|c} 
Amplitud [\%] & Precisión [\%] & S \\
\hline 20 & 96.40 & 0.084 \\
40 & 93.90 & 0.116 \\
60 & 95.52 & 0.130 \\
80 & 94.35 & 0.113
\end{tabular}

\subsection{RESULTADOS PRUEBA DINÁMICA}

Los resultados aquí mostrados han sido obtenidos de un test desarrollado en un circuito simulando situaciones reales de conducción [8]. El periodo de muestreo empleado es de $1 \mathrm{~ms}$, lo que prueba su aplicabilidad y eficiencia para tiempo real.

El resultado de la respuesta del freno se muestra en la Fig. 11, donde se muestra como la simulación sigue con precisión la respuesta real. En la Fig. 12 se muestra la diferencia entre ambas curvas, siendo el error absoluto menor que $2.5 \%$ para todo el recorrido, con un error medio de 0,59\%. Para calcular este valor, se han omitido los tramos donde ambas señales son cero, para no dañar el cálculo.

En cuanto al resultado del volante, en la Fig. 13 se muestra su respuesta a lo largo de todo el recorrido. Como se puede observar, la simulación también ofrece una precisión muy alta. Aunque, como

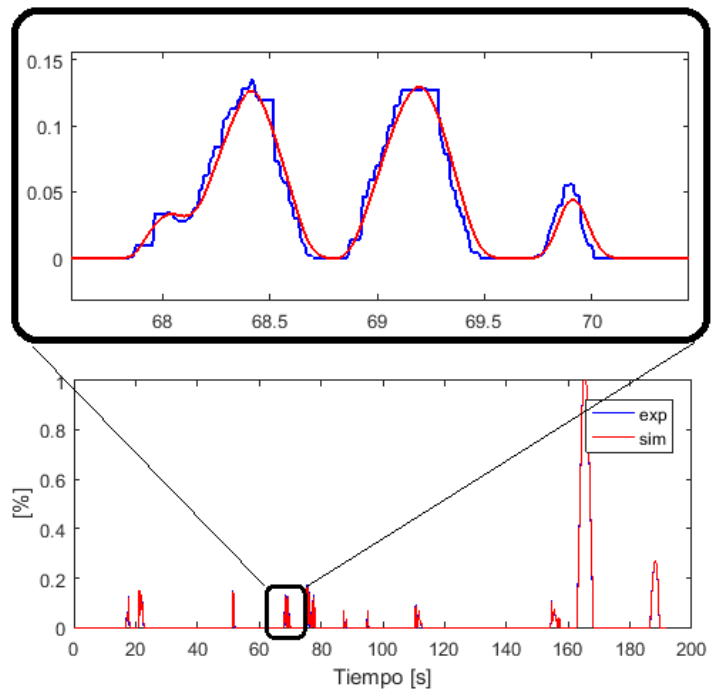

Figura 11: Respuesta del actuador del freno

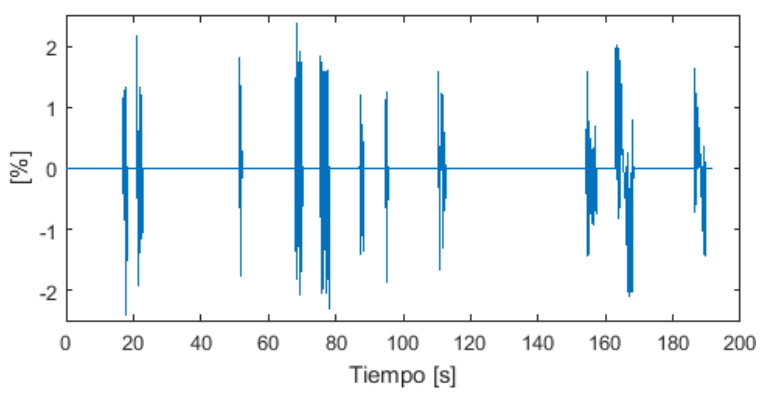

Figura 12: Error de la simulación del freno

se ve en la Fig. 14, el error máximo asciende a picos del $12 \%$. Esto está causado debido a que al desarrollar el modelo, el punto de operación no excedía un rango superior a $\pm 40 \%$. Por lo que en los giros de mayor amplitud, el error aumenta. A pesar de ello, el error medio cometido es de $\pm 1,10 \%$.

\section{CONCLUSIONES}

En este documento se han desarrollado dos metodologías diferentes para obtener el modelo matemático de un actuador. Para el sistema de frenado, la aplicación System Identification Tool resulta muy útil, ya que ofrece la posibilidad de trabajar con sistemas en forma de caja negra, donde no es necesario conocer una planta para poder modelarla. A pesar de ello, esta aplicación está orientada a modelar sistemas reales con dinámicas simples, mientras que el sistema modelado se trata de un conjunto de motor más controlador. Por ello, resulta necesario efectuar ciertas modificaciones al modelo obtenido para obtener una mayor precisión. En cuanto a su punto de funcionamiento, 

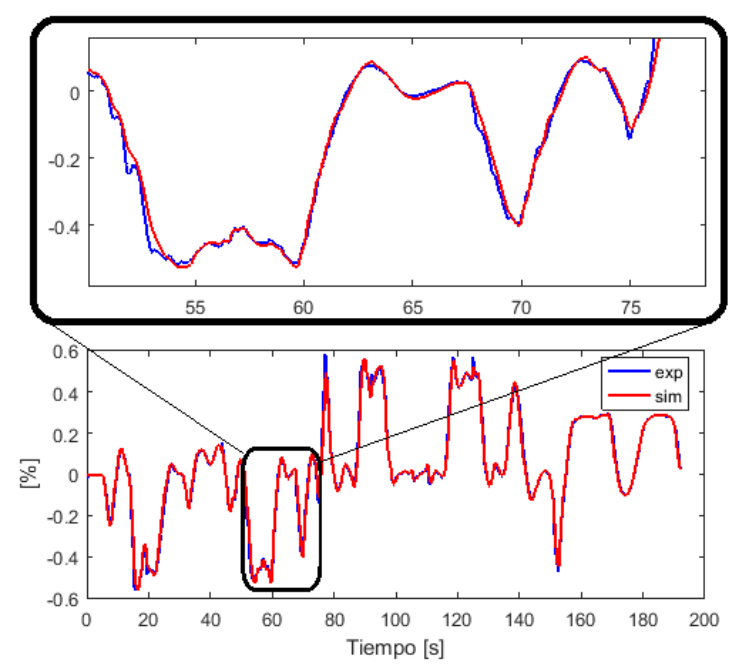

Figura 13: Respuesta del actuador del volante

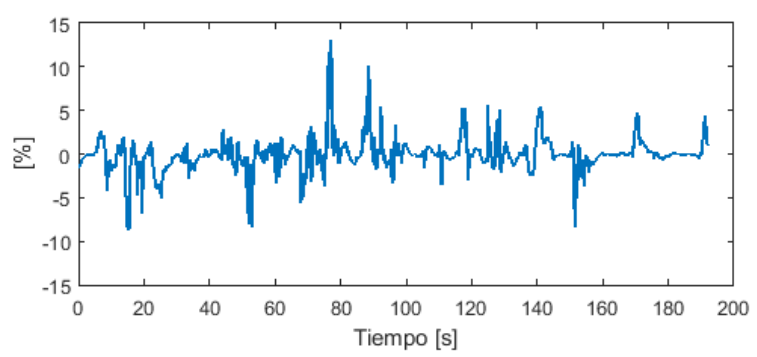

Figura 14: Error de la simulación del volante

cabe destacar que la amplitud predominante en las pruebas realizadas es alrededor del $15 \%$ de la señal, por lo que el modelo deberá ser más preciso en torno a ese valor. En lo referente al sistema de dirección, se ha obtenido un modelo satisfactorio con el simple conocimiento de los parámetros del motor, sin necesidad de saber los parámetros del controlador instalados en el vehículo. En la estimación del modelo desarrollada a partir de las pruebas estáticas, estas están limitadas a una amplitud de giro inferior al $40 \%$. Esto se debe a que para valores superiores, la fricción de las ruedas en parado ofrece una resistencia excesiva, pudiendo dañarse la integridad del motor. En la prueba dinámica, en cambio, no existe dicho inconveniente, dado que al estar el vehículo en movimiento las fuerzas en reacción al giro se ven disminuidas. Por ese motivo el modelo debe extrapolar la respuesta para rangos superiores. En cuanto a la validación, los modelos de los actuadores ejercen un papel fundamental en la simulación del vehículo. Un error mayor al $5 \%$ aquí puede provocar una trazada en la trayectoria totalmente diferente en el vehículo. En este caso, los modelos obtenidos ofrecen una respuesta muy similar a la del vehículo real, con un error muy bajo, lo que muestra la similitud entre la respuesta real del vehículo y la respuesta del simulador. Por ello, el simulador es válido como una herramienta precisa para realizar pruebas.

\section{English summary \\ LOW LEVEL CONTROL ACTUA- TED SYSTEM MODELLING AP- PROACH FOR HIGHLY AUTO- MATED VEHICLES}

\begin{abstract}
Automated driving requires proper simulation models for the research and development of decision and control strategies. Currently, real testing platforms are obtained through the modification of comercial vehicles, where electro-mechanical actuators or robots are fixed to the steering wheel or gas/brake pedals. During the setting of the gains of high level control, remarkable differences may occur between reality and simulation due to improper models of the low level control architecture. In this project, a wide overview about the state-of-art of the low-level control techniques used not only in academic papers, but also in the industry, is presented. Aditionally, a validation methodology for the low level actuator system model is developed based on openloop trials. Finally, a comparison between real and simulated behaviours of the modelled actuator system is carried out, analysing the performance of the proposed methodology in a real case scenario with an automated vehicle.
\end{abstract}

Keywords: Automated driving, parameter identification, modeling, simulating, electro-mechanic actuators, automation.

\section{Referencias}

[1] Pérez, J., Godoy, J., Villagrá, J., \& Onieva, E. (2013, May). Trajectory generator for autonomous vehicles in urban environments. In 2013 IEEE International Conference on Robotics and Automation (pp. 409-414). IEEE.

[2] AEVIT, Advanced Electroni Vehicle Intereface Technology, Owners Manual, USA. 
[3] Darpa Urban Challenge - The book, (2007),(vol. 56), USA: Springer.

[4] Tas, O.Bandera, C., René, M., Poggenhans, F., Salscehider, N. O., et al. (2018) IEEE Transactions on intelligent transportation systems,Bertha-Team Annieway 2016 Grand Cooperative Driving Challenge (vol. 19)

[5] Sell, R., Rassolkin, A., Leier, M., Ernits, J., (2018), Self-driving car ISEAUTO for research and education, Delf, Paises Bajos.

[6] Oguntoyinbo, J., (2009),PID of Brushless DC motor and robot ttrajectory planning and simulation with Matlab/Simulink, Finland.

[7] Ljung, L., (2002) Prediction error estimation methods, Circuits, Systems and Signal Processing, (vol. 21).

[8] Matute-Peaspan, J. Marcano, M., Diaz, S., Pérez, J. (2019). Experimental Validation of a Kinematic Bicycle Model Predictive Control with Lateral Acceleration Consideration In 10th IFAC Symposium on Intelligent $\mathrm{Au}-$ tonomous Vehicles. Poland

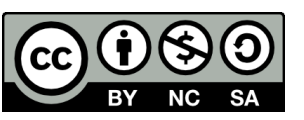

C 2019 by the authors. Submitted for possible open access publication under the terms and conditions of the Creative Commons Attribution CC BY-NC-SA 4.0 license (https://creativecommons.org/licenses/by-ncsa/4.0/deed.es). 